\title{
Lesion recognition by bifunctional DNA-glycosylase Endo III and its catalytic mutants
}

\author{
O.A. Kladova ${ }^{1 *}$, N.A. Kuznetsov ${ }^{1,2}$, O.S. Fedorova ${ }^{1,2}$ \\ ${ }^{1}$ Institute of Chemical Biology and Fundamental Medicine SB RAS, Novosibirsk, Russia \\ ${ }^{2}$ Novosibirsk State University, Novosibirsk, Russia \\ *e-mail:kladova@niboch.nsc.ru
}

Key words: base excision repair, structural transitions, thermodynamic analysis, pre-steady state kinetics

Motivation and Aim: Endonuclease III is a bifunctional DNA-glycosylase from E. coli that exhibits both $\mathrm{N}$-glycosylase and AP-lyase activity. It is known that interaction of enzyme with DNA substrate proceeds through several conformational rearrangements in its structure. Here we used stopped-flow technique to register conformational dynamics of DNA substrates arising from the Endo III action.

Methods and Algorithms: Structural changes in DNA substrates were registered using fluorescent analog of DNA base incorporated opposite the specific base. The DNA duplexes contained 5, 6-dihydrouracile base, non-cleavable analog of abasic site and native guanosine as non-damaged DNA-duplex. Changes in fluorescence intensity were registered in $5-37^{\circ} \mathrm{C}$ temperature range. To calculate the rate constants of DNA conformational transitions, a number of kinetic curves for each DNA substrate were obtained. Additionally, several mutant forms (K120A, D138A) were constructed to determine the functional role of each amino acid residue in the catalytic process.

Results: It was shown that mutant forms Endo III K120A and D138A lack of both $\mathrm{N}$-glycosylase and AP-lyase activities. Understanding the mechanism of the catalytic complex formation was improved by thermodynamic analysis of the binding and cleavage steps. The dependences of equilibrium constants on temperature were analyzed according to the van't Hoff equation allowing calculating Gibbs free energy, enthalpy and entropy of individual interaction stages. The analysis of the temperature dependence of the reaction rate constant $\mathrm{k}_{\text {cat }}$ using the Eyring equation provided the values of standard activation enthalpy and standard activation entropy of the transition state.

Conclusion: The thermodynamic analyses of catalytic processes performed by Endo III from E. coli reveal that this enzyme employs common energetic features at the main steps of base lesion recognition as other DNA-glycosylases from human and bacteria. Mutational analysis of Endo III reveals that Lys120 takes part both in the processes of nonspecific binding and subsequent recognition of the damage because substitution K120A significantly decelerates conformational changes of the duplex during the complex formation. Moreover, substitution D138A causes a complete loss of the ability of Endo III to distort a DNA double chain.

Acknowledgements: This work was supported partially by Russian State funded budget project (VI.57.1.2, 0309-2018-0001) and grant from the Russian Foundation of Basic Research (16-04-00037). 